\title{
Multiple Comparisons of Traditional Acupuncture Therapies of Japan, Korea and China-A Preliminary Report of Three Countries' Acupuncture (TCA) Project
}

\author{
Kenji Kawakita ${ }^{*}$, Yong-Suk Kim², Nobuo Yamaguchi ${ }^{3}$, Xiao-Pin Lin ${ }^{4}$, Matsuo Arai ${ }^{4}$, \\ Naomi Takazawa4, Shan-Yu Su ${ }^{5}$ \\ ${ }^{1}$ Department of Physiology, Meiji University of Integrative Medicine, Kyoto, Japan \\ ${ }^{2}$ Department of Acupuncture \& Moxibustion, (Brain \& Neurological Disorders and Pain), Kangnam Korean \\ Hospital, Kyung Hee University, Seoul, Korea \\ ${ }^{3}$ Kanazawa Medical University, Ishikawa, Japan \\ ${ }^{4}$ Ishikawa Natural Medicinal Products Research Center, Kanazawa, Japan \\ ${ }^{5}$ School of Post-Baccalaureate Chinese Medicine, College of Chinese Medicine, China Medical University, \\ Taichung, Taiwan \\ Email: ${ }^{*}$ k kawakita@meiji-u.ac.jp
}

Received 14 May 2015; accepted 25 August 2015; published 28 August 2015

Copyright (C) 2015 by authors and Scientific Research Publishing Inc.

This work is licensed under the Creative Commons Attribution International License (CC BY).

http://creativecommons.org/licenses/by/4.0/

(c) () D Den Access

\section{Abstract}

Acupuncture therapy had established in China and it distributed among Asian countries and developed in each country as traditional medicine. In this project we compared the procedures and effects of traditional acupuncture therapies in China, Korea and Japan by using 10 healthy young male volunteers with written informed consent. Total white cell counts, leukocyte subsets and noradrenalin contents were used as outcomes. Three well trained traditional acupuncture therapists were chosen and they treated the same subjects at random order with intervals of at least two months. Each acupuncturist made his diagnosis and points selection for potentiate vital conditions. Clearly different procedures and point selections were used among three traditional acupuncture therapies on the same subject and the obtained changes of outcome measures also differed. Although variations of data were quite large, these results clearly demonstrated that acupuncture therapies have developed among three countries in unique manner.

\section{Keywords}

Acupuncture, Total Leukocyte, Leukocyte Subset, Emotional Hormone, QOL, Comparison Among

\footnotetext{
"Corresponding author.
}

How to cite this paper: Kawakita, K., Kim, Y.-S., Yamaguchi, N., Lin, X.-P., Arai, M., Takazawa, N. and Su, S.Y. (2015) Multiple Comparisons of Traditional Acupuncture Therapies of Japan, Korea and China-A Preliminary Report of Three Countries' Acupuncture (TCA) Project. Open Journal of Immunology, 5, 79-89. http://dx.doi.org/10.4236/oji.2015.53009 


\section{Introduction}

It is widely accepted that acupuncture and moxibustion therapies are originated from China, and it has grown and established its own system as traditional medicine, then it distributes to worldwide as traditional Chinese Medicine (TCM). The diagnosis and procedure of TCM have been well established and recently various reevaluation of TCM has been conducted [1]-[4]. The majority of practitioners have learned acupuncture therapy in China or Chinese doctors in the different countries, so TCM way of acupuncture therapy may be considered as only one acupuncture therapy in the world.

During the process of development and worldwide distribution, it has modified in different countries with their nature, social customs, foods and other things. Thereafter the term "acupuncture and moxibustion therapies" includes several different procedures of diagnosis and treatment in them, although existence of the differences has not been hardly noticed the researcher of clinical trials of acupuncture in the Western world.

In Korea and Japan, acupuncture therapy has developed as one of traditional therapies with different characteristics from that of TCM. Korean acupuncture has developed on the basis of "Sasang" constitutional medicine, and Saam acupuncture has established as Korean particular way of diagnosis and treatment procedures of acupuncture [5] [6]. Sasang constitutional medicine is characterized as holistic tailored medicine [7], and its reliability of a questionnaire for the diagnosis is confirmed recently [8].

In Japan, acupuncture therapy has long been used as the main stream medicine until Edo era (the middle of 19 century), and it is usually characterized by its use of needling tube with fine needles and gentle manipulation without de-qi sensation [9]-[11]. In our previous multi-center trial, gentle manipulation to the specific point at the throat is used as acupuncture for prevention of the symptoms of common cold [12]. In practical situation, wide variation of diagnosis and needling techniques are used such as meridian, reaction point, trigger point, skin impedance, anatomy-physiology, and so on. These variations of Japanese acupuncture may due to its position of the medical systems in Japan. It has developed for the requirement of the patients. Recently, more modernized acupuncture based on physiology and anatomy of the body has been developed to cooperate with Western medical doctors.

On the other hand, recent clinical trials of acupuncture have provided an important evidence of efficacy and safety of acupuncture treatment, and the majority of acupuncture interventions used in the trials is based on TCM theory of acupuncture points selection and manipulations [13]. The mega trials conducted in German clearly demonstrate that real TCM style acupuncture has a statistically superior effect compared with conventional and/or standardized Western therapies [14]. The economic analysis also demonstrates the cost-benefit of acupuncture therapy in German acupuncture trials [15].

The major issues to be considered now is that there is no statistically significant difference between real TCM acupuncture group and those of sham acupuncture (minimal acupuncture) group, that is no specific effect of acupuncture therapy was proven by high quality RCTs conducted in German. In the trials, minimal acupuncture is characterized by shallow needling to non-acupuncture points without de-qi sensation. As the majority of researchers of clinical trials of acupuncture in the Western world have learned and trained TCM as standard acupuncture therapy, so the location of acupuncture points and provocation of particular de-qi sensation is considered to be essentially important. So the German trials choose the minimal acupuncture as sham intervention might be reasonable.

However, the shallow needling without de-qi used in minimal acupuncture have been widely used in Japan and selection of acupuncture points tended to be based on careful palpation of the skin and/or deep tissues not restricted at the precise location in textbook [16]. In other words, the minimal acupuncture is not adequate intervention as inert sham intervention because it has been used in Japan as one of the procedure of real acupuncture manipulation.

Therefore it is important to know the existence of different procedures of acupuncture except for TCM acupuncture therapy, however, no clinical researches comparing various traditional acupuncture therapies have been conducted in evidence based manner so far.

Our previous study clearly demonstrated that one session of acupuncture treatment by Japanese acupuncture, called Tai-chi (太極) therapy, one of traditional acupuncture therapies could induced remarkable effects on im- 
mune system [17]. Then Prof. Yamaguchi proposed a project called “銊三国師 (TCA: three countries” acupuncture) project” to investigate the different therapeutic properties of traditional acupuncture in Japan, Korea and China. The protocol of the present project conducted was determined through several meetings and discussions among the researcher in China, Korea and Japan.

The purpose of TCA project is to clarify the different characteristics of traditional acupuncture procedures in three countries and it may help to understanding and development of potential roles of acupuncture therapy in the future CAM.

\section{Methods}

\subsection{Subjects and Practitioners}

Subjects were healthy young subjects with informed consent (male, average 21 years, $20-25, n=10$ ). They had no experience of acupuncture treatment. The experimental design used was cross-over and each subject was randomly allocated to Chinese, Korean and Japanese acupuncture in order of their registration. One subject received three different treatments. The experiments were conducted in April, June and August in 2009 at Kanazawa.

Acupuncture practitioners were well-experienced traditional acupuncture therapists in Japan, Korea and China (their clinical experiences were over 20 years). The practitioners were asked to make diagnosis on each subject by their own methodology and selected best acupuncture points for individualized treatments in each subject to improve their conditions based on their diagnosis. The purpose of the treatment was to enhance self-defense system and to make better health condition in each subject. The concrete procedures of diagnosis and treatment were recorded on the chart in detail, and the processes of diagnosis and treatment were recorded by video camera with permission of the subjects and practitioners.

The subjects were treated by three practitioners in three different styles of acupuncture treatments at intervals of about 2 months or more. The experiments usually started in a clinic at 19:00 in each time to avoid the circadian changes in adrenalin contents and white blood cells [18]. The experiments were conducted on April, June and August. An additive experiment by Chinese acupuncture treatment was performed on January 2010 as the initial Chinese practitioner was accidentally exchanged and the first three subjects data collected in April was replaced by the new samples. Three beds were prepared and each practitioner treated three subjects in each experimental session. The average treatment time was about 30 mins. The procedures of diagnosis and treatments were recorded by three video cameras with permission of the subjects and practitioners.

The written informed consents were obtained, and the protocol of this project was approved by the Ethics Committee of Kanazawa Medical School.

\subsection{Measurement of White Blood Cell Composition and Adrenalin Contents}

Blood samples were collected by nurse before acupuncture treatment and 24 hours after the treatment. The blood samples were analyzed conventional procedures and measured the total leukocytes (WBC: white blood cell) number and ratio of granulocytes and lymphocytes. The catecholamine content (adrenalin) was also measured by ELISA (Enzyme-Linked Immuno Sorbent Assay).

\subsection{Statistical Analysis}

The data were expressed as mean $+/-$ standard deviation. The WBC is number of cells, granulocytes and lymphocytes were shown as \% of total leukocytes and adrenalin content was expressed by pg (pikogram $/ \mathrm{ml}$ ). Group comparison of data was performed by ANOVA and post hoc multiple test. Difference of point selection between two countries was analyzed by chi-square test (Statview, 5.0, SAS). The correlation between baseline data and ratio of each WBC composition and adrenalin were also calculated.

\section{Results}

\subsection{Diagnosis and Treatment of Three Countries}

Subjects included in this pilot project were ten healthy young male university students, and they were diagnosed by three traditional acupuncturists in each treatment session and point selections were performed by individua- 
lized manner. Korean acupuncturist clearly classified based on Sasang constitution theory, a traditional Korean medical theory. Nine subjects were classified into Soeum-in type $(n=4)$, Soyang-in type $(n=1)$, Taeum-in type $(n=3)$ and Tayang-in type $(n=0)$ by Sasang constitutional medicine, and deficiency of responsible meridians were pointed out. The point selection was conducted by base on the Saam acupuncture theory, and the points located in the periphery were frequently used. Supplement or drain technique on the selected points were restrictedly performed.

Chinese acupuncturist used pulse and tongue diagnosis then selected the points. No TCM diagnosis of condition was performed as the subjects were basically healthy young students. The relatively thick acupuncture needles were used and inserted deep in tissues and de-qi sensation frequently provoked by the needle manipulation.

Japanese acupuncturist did careful palpation of the skin and deep tissues to detect reaction points, no pulse diagnosis was used. The needling procedure was gentle manipulation without any subjective sensation called deqi. He chose the points regardless of the precise location of acupuncture points so no information regarding selection of the acupuncture points used was available but rough location of needling points was recorded. The extremely different characteristic of the Japanese style acupuncture from that of $\mathrm{C}$ and $\mathrm{K}$ treatment is apparent.

All practitioners used the single-use disposable acupuncture needle in sterile package.

\subsection{Point Selection of Korean and Chinese Acupuncturists}

Table 1 summarizes the acupuncture points selected by Korean and Chinese practitioner in all subjects of this study. The total numbers of acupuncture points used were similar but the actual points used were quite different between $\mathrm{C}$ and $\mathrm{K}$ treatments. Total number of acupuncture points used in C and $\mathrm{K}$ treatment were 83 (4 - 17, mean of 9.2/subject) and 75 (6 - 9, mean of 8.3/subject), and less and regular number of points selection were observed in $\mathrm{K}$ treatment.

The acupuncture points used in $\mathrm{C}$ and $\mathrm{K}$ treatments were obviously different, and only 5 acupuncture points (ST36, V12, LI11, LR3, SP2) were used in both K and C treatment, although the subjects were the same and a total of 44 acupuncture points were used in $\mathrm{K}$ and $\mathrm{C}$ treatment. The major points used in $\mathrm{C}$ and $\mathrm{K}$ treatments were listed in Table 2.

\section{WBC Number}

The total WBC number, lymphocyte (\%), granulocyte (\%), serum adrenalin content (pg/ml) were measured before treatment as baseline. The baseline data of nine subjects who complete three acupuncture therapies received were listed in Table 3.

One subject was dropout for his personal reason. There was no significant difference among three groups (China, Korea and Japan) in their baseline data although individual variations tended to be large.

\section{WBC Counts}

The effects of $\mathrm{C}, \mathrm{K}$ and $\mathrm{J}$ acupuncture treatment on the total number of WBC counts were summarized in Figure 1 and Figure 2. Relative changes in percentage compared with the baseline (before treatment) were indicated as

Table 1. Point selection by K and C practitioners.

\begin{tabular}{cccc} 
& points & range & mean \\
\hline China & 83 & $6-9$ & 9.22 \\
Korea & 75 & $4-17$ & 8.33 \\
\hline
\end{tabular}

Table 2. Rank of the acupuncture points frequently used in $\mathrm{K}$ and $\mathrm{C}$ treatments.

\begin{tabular}{ccccccrr}
\hline Points & 1 & 2 & 3 & 4 & 5 & 7 & 6 \\
\hline China & BL23 & ST36 & SP6 & CV4 & KI3 & GV4 \\
Korea & SP3 & CV12 & ST36 & LI4 & SI2 & HT7 \\
\hline
\end{tabular}




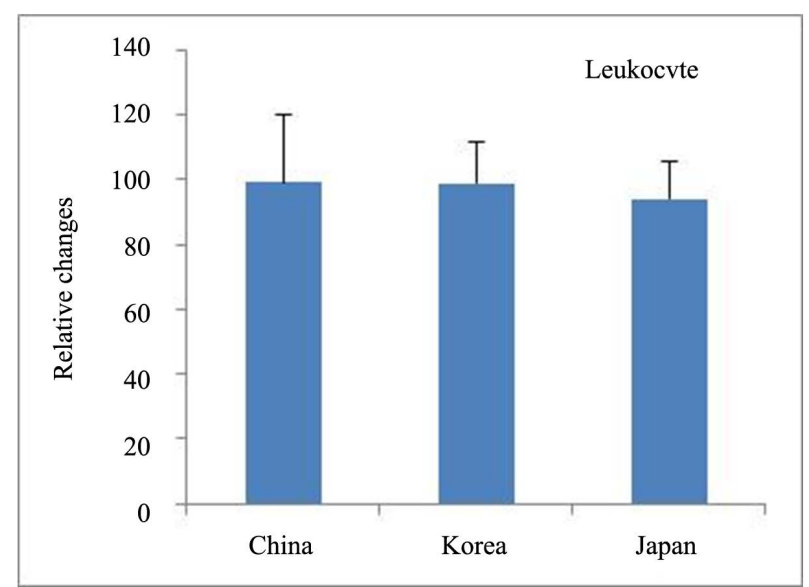

Figure 1. Changes of WBC numbers (\% of baseline) after one acupuncture session.

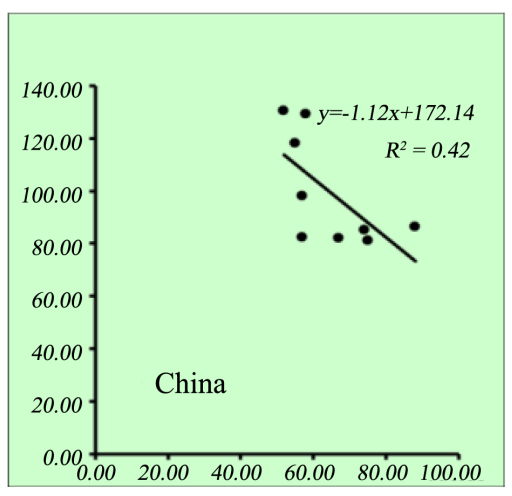

(a)

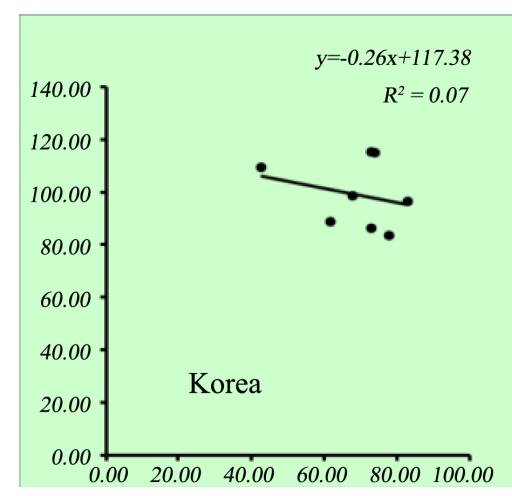

(b)

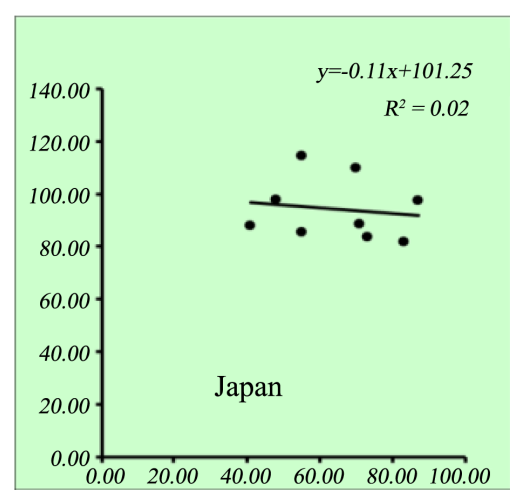

(c)

Figure 2. (a)-(c) Relations between the baseline data and percent change after acupuncture treatments. Vertical scale: relative changes in leukocyte number 24 hrs after treatment. Horizontal scale: baseline data of leukocyte number $\left(\times 100 / \mathrm{mm}^{3}\right)$.

Table 3. Baseline data of leukocytes (number *100), lymphocytes (\%), Lymphocytes(\%) and serum adrenalin (pg/ml).

\begin{tabular}{|c|c|c|c|}
\hline & mean & SD & $n$ \\
\hline \multicolumn{4}{|c|}{ Leukocytes } \\
\hline China & 64.78 & 12.04 & 9 \\
\hline Korea & 69.25 & 12.30 & 8 \\
\hline Japan & 64.78 & 15.80 & 9 \\
\hline \multicolumn{4}{|c|}{ Lymphocytes } \\
\hline China & 39.91 & 9.49 & 9 \\
\hline Korea & 31.21 & 7.23 & 8 \\
\hline Japan & 37.12 & 10.03 & 9 \\
\hline \multicolumn{4}{|c|}{ Granulocytes } \\
\hline China & 55.54 & 9.61 & 9 \\
\hline Korea & 64.76 & 8.29 & 8 \\
\hline Japan & 58.98 & 11.26 & 9 \\
\hline \multicolumn{4}{|c|}{ Adrenalin } \\
\hline China & 51.67 & 21.05 & 9 \\
\hline Korea & 57.00 & 16.21 & 8 \\
\hline Japan & 61.78 & 29.04 & 9 \\
\hline
\end{tabular}


columns and vertical bars (mean and SD) in Figure 1.

Total WBC counts did not change after the treatment and slight decrease in $\mathrm{J}$ treatment is observed. The largest SD in $C$ treatment indicates the strong influence of $C$ treatment on WBC counts. No significant differences were observed (ANOVA, $p=0.749$ ). Figures 2(a)-(c) summarizes the relations between the baseline data and degrees of changes in percentages after three treatments. All subject's data were plotted and regression lines in three treatments was shown in each figures. In C treatment, negative slope of regression line indicates the higher baseline WBC subjects tended to be reduced by the treatment. In K and J treatments, no such tendencies could be observed, although the individual variations tend to be large (small coefficients of determination).

\section{Lymphocyte Ratio}

The effects of $\mathrm{C}, \mathrm{K}$ and $\mathrm{J}$ treatments on the lymphocytes were summarized in Figure 3 and Figure 4 Influences of acupuncture treatments on lymphocytes differ among the countries. In $\mathrm{C}$ treatment tended to decrease the relative value to $91.1 \%$, whereas $\mathrm{K}$ and $\mathrm{J}$ treatments increase to 109.5 and $108.6 \%$, although no statistical significance observed (one way ANOVA, $P=0.0784$ ). The variation of SD among the countries is similar.

Figure 4 summarizes the relation between the baseline data and degree of changes in percentages of lymphocytes ratios after the treatment. In $\mathrm{C}$ and $\mathrm{J}$ treatments, negative regression lines, whereas $\mathrm{K}$ treatment produce

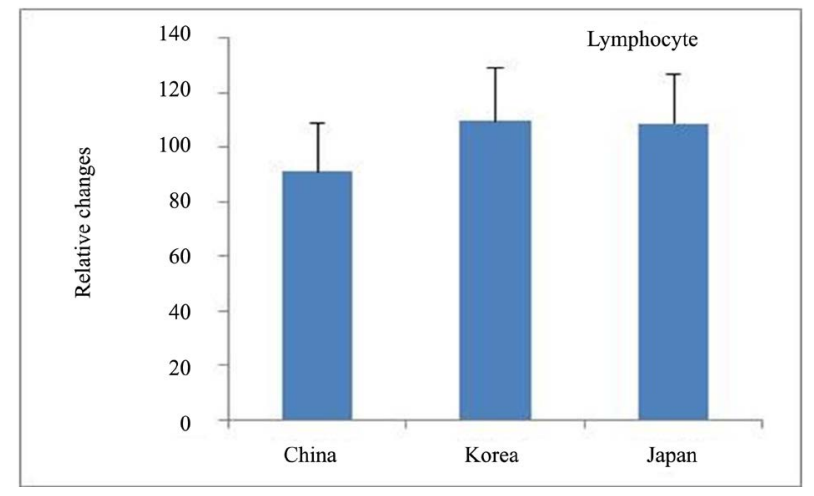

Figure 3. Changes of Lymphocyte ratios (\% of baseline) after acupuncture session.

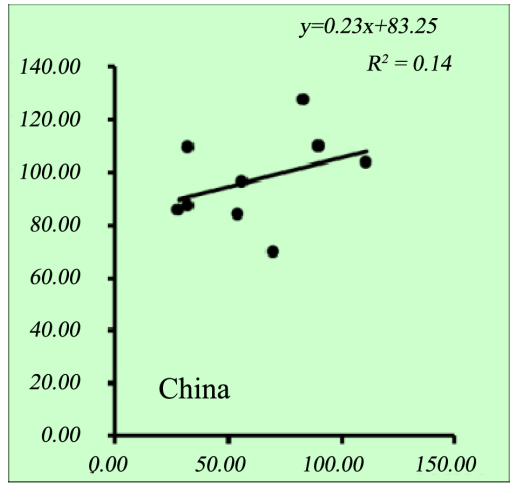

(a)

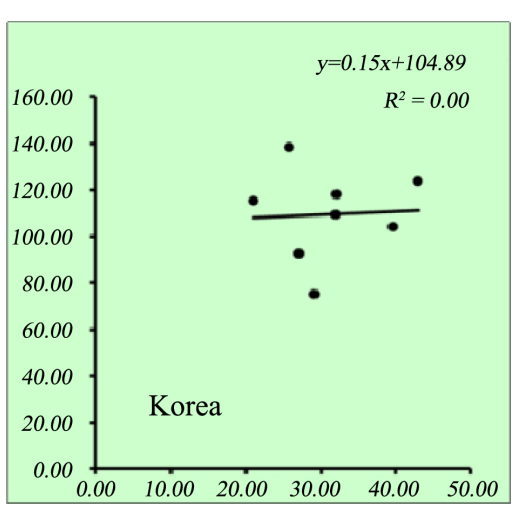

(b)

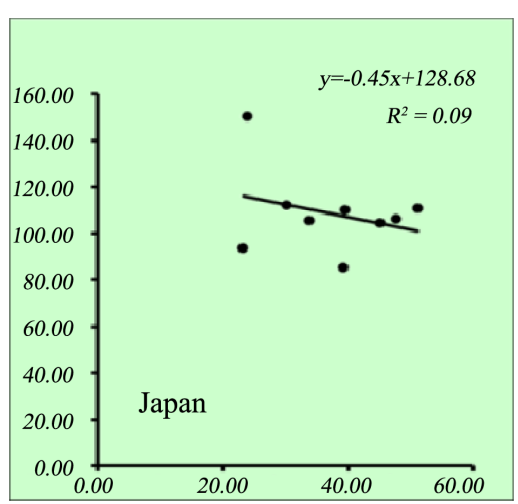

(c)

Figure 4. (a)-(c) Relations between the baseline data and percent change after acupuncture treatments. Vertical scale: relative changes in lymphocytes number 24 hrs after treatment. Horizontal scale: baseline data of ratios of lymphocytes (\%).

slight positive regression.

\section{Granulocyte Ratio}

The effects of $\mathrm{C}$, $\mathrm{K}$ and $\mathrm{J}$ treatment on the ratio of lymphocytes were summarized in Figure 5 and Figure 6. Effects of acupuncture treatments on granulocytes ratios are shown in Figure 5. 
On granulocytes ratios are basically opposite pattern of those of lymphocyte. Only C treatment increased to $108.8 \%$ and $\mathrm{K}$ and $\mathrm{J}$ treatments reduced to 95.1 and 95.5 , respectively. There are significant difference among the countries (ANOVA, $p=0.026$ ).

The negative slope of regression lines was observed in $\mathrm{C}$ treatments and positive slopes were obtained in $\mathrm{K}$ and $\mathrm{J}$ treatments.

\section{Changes in Adrenaline Contents}

Relative changes in adrenalin contents are shown in Figure 7 and Figure 8. Regarding adrenalin contents, three treatments have different influences. In $\mathrm{C}$ treatment, increase of the contents with large variation in $\mathrm{C}$ treatment whereas slight reduction with small variation in $\mathrm{J}$ treatment. $\mathrm{K}$ treatment has no changes as the mean value although its variation is large,

Relative changes of adrenalin contents after acupuncture treatments are shown as a regression lines in Figure 8. The negative slopes of regression lines in $\mathrm{C}$ and $\mathrm{K}$ treatments are observed whereas a positive line in $\mathrm{J}$ treat-

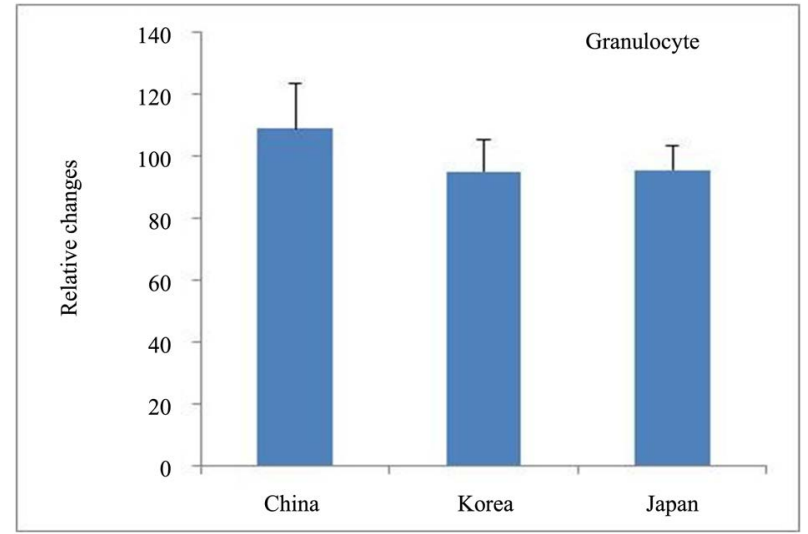

Figure 5. Changes of Granulocyte ratios (\% of baseline) after one acupuncture session.

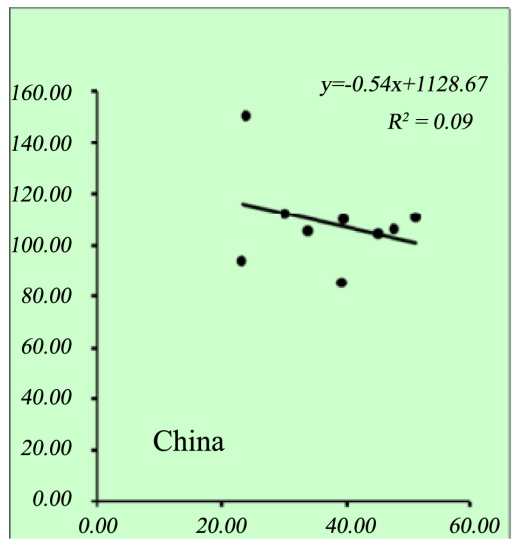

(a)

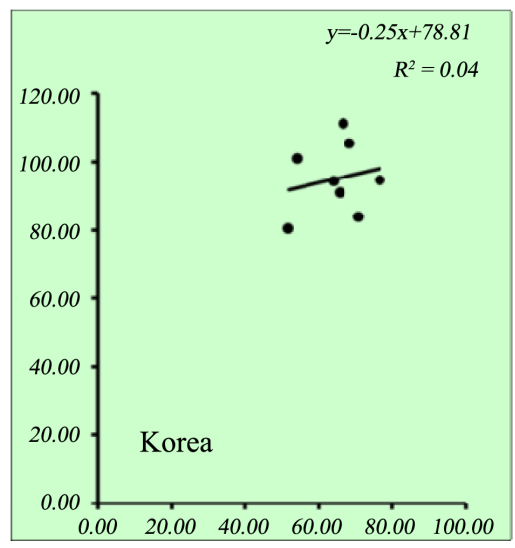

(b)

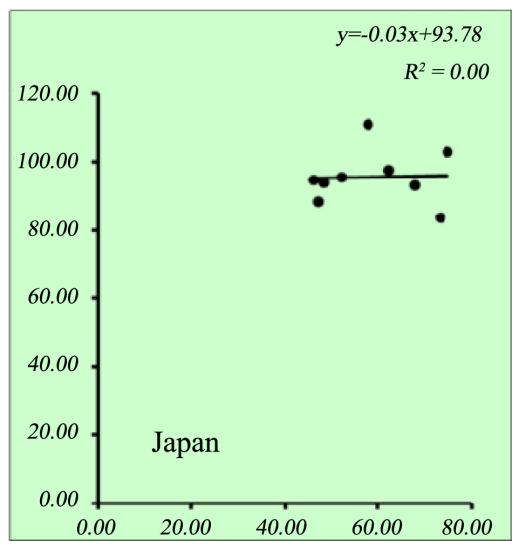

(c)

Figure 6. (a)-(c) Relations between the baseline data and percent change after acupuncture treatments. Vertical scale: relative changes in leukocyte number 24 hrs after treatment. Horizontal scale: baseline data of ratios of granulocytes (\%).

ment is detected. These patterns of regression lines are different from those observed WBC and their subclasses (granulocytes and lymphocytes).

\section{Discussion}

In the present pilot study, we compared the effects of three different individualized diagnoses and treatments of 


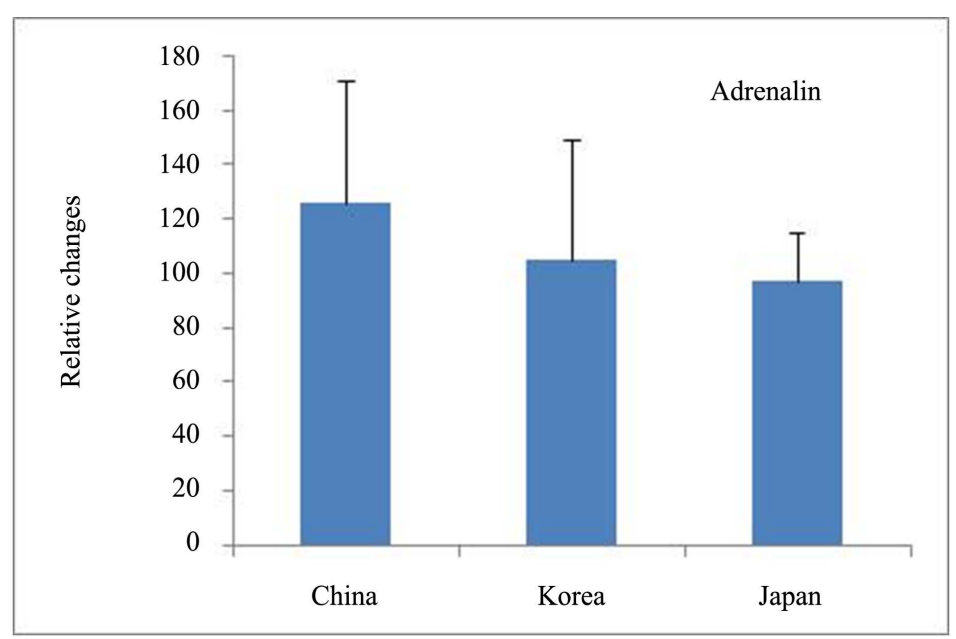

Figure 7. Changes of Adrenalin contents (\% of baseline) after one acupuncture session.

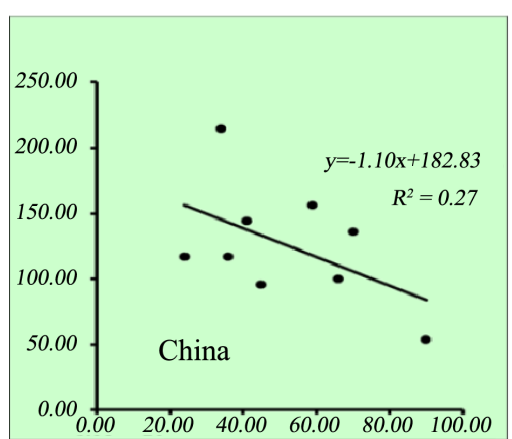

(a)

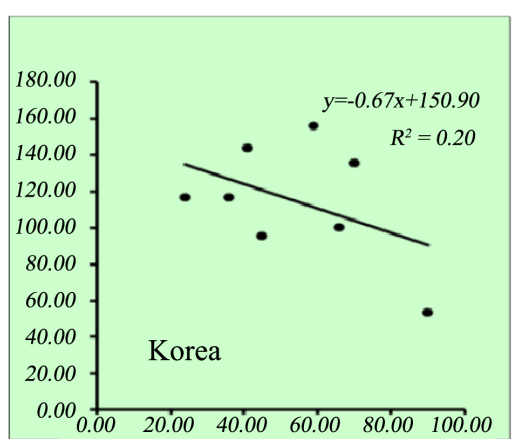

(b)

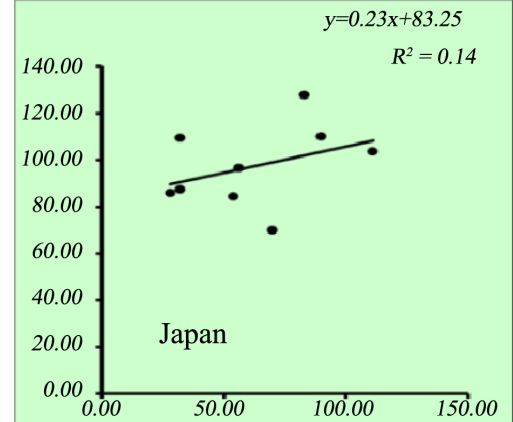

(c)

Figure 8. (a)-(c) Relations between the baseline data and percent change after acupuncture treatments. Vertical scale: relative changes in leukocyte number 24 hrs after treatment. Horizontal scale: baseline data of serum adrenalin contents (pg/ml).

traditional ways of Chinese, Korean and Japanese acupuncture on the WBC composition and adrenalin in the same subject. The apparently clear differences of point selection and procedure of acupuncture manipulations among the countries were observed. A total of 44 acupuncture points was selected but only 5 points were common in $\mathrm{K}$ and $\mathrm{C}$ treatments. Three different acupuncture treatments also induced different tendencies in modification of the WBC counts and lymphocyte and granulocyte ratios and serum adrenalin although no statistically significant as obvious individual variations also observed.

The facts that multi-directional changes in the outcome measures could be produced by three different acupuncture treatments suggested the importance of procedures of acupuncture needling. In this study, Chinese acupuncture produced de-qi sensation, and Korean acupuncture frequently used the acupuncture points in the hand. On the contrary, Japanese acupuncture provoked almost no subjective sensation but the practitioner detected slight changes in respiratory movement during his needling technique. These three acupuncture treatments undoubtedly induced several modifications on the immune and autonomic nervous systems in different manner.

The immune system was strongly influenced by various factors. Psychological state such as depression and mental stress suppressed the immune activities [19] Acupuncture and moxibustion treatment could improve and modify the immune activity by measuring cytotoxicity of lymphocytes and NK cell count [20]-[22]. On the contrary, lack of effect of acupuncture on the immune modulatory effects was also reported [23].

Recently analysis of gene by using cDNA microarray analysis demonstrated participation of several genes which related to the up-regulation of NK cell activity by electro-acupuncture (EA) [24]. Down-regulation of proinflammatory cytokine such as IL-I beta mRNA by EA was also shown [25]. 
In mice, EA activated the spleen NK cells and IFN-gamma level also increased, and EA also increased beta endorphin level and its effects were antagonized by naloxone [26]-[29].

Anti-inflammatory effect of acupuncture was suggested to be mediated by vagal nerve stimulation and deactivation of macrophages [21], and the fact that existence of acetylcholine receptors on the lymphocytes [30] suggested the importance of tune of autonomic nervous system on the immune activity.

Our previous study clearly demonstrated that one of Japanese-style acupuncture treatment induces prolonged increases of cytokines and shift of ratios of granular cells and lymphocytes depending on the previous condition of the subjects. The increases of IFN-gamma and IL-I6 and other leukocytes with various CD marker including NK cells were observed. These promoting influences of acupuncture on the immune systems and reciprocal effects on lymphocytes and granulocytes might be a possible explanation of bio-regulatory function of acupuncture [18] [30] [31]. Then measurement of ratios of the lymphocytes and granulocytes proposed as a useful outcome measures in complementary and alternative (CAM) medicine [17].

In the present study no such changes in lymphocytes and granulocyte was observed in the subgroup analysis of G-type and L-type subjects, although the same acupuncture practitioner with the previous study participated in this project.

The large variation of baseline data in small sample size might be a possible cause of the discrepancy of results. Health conditions of the subjects, young university students, varied during the relatively long periods (4 months) accompanied with seasonal changes of circumstances, so the present data could not be assumed as the simple results of $\mathrm{J}$ treatment. The similar problems were also found in the characteristics of the modulating potentials of $\mathrm{K}$ and $\mathrm{C}$ acupuncture treatments. Another issue was the subject used in the present study. The traditional acupuncture procedures and its theoretical concepts might developed based on their subjects in each country, so the facts that the subjects in this study were Japanese young students might also be a possible cause of large variation of the results in $\mathrm{K}$ and $\mathrm{C}$ treatments.

The results obtained in the present study were not conclusive but we got important information about the variety of acupuncture treatments in Japan, Korea and China, and they have potentials to modulate the immune systems and stress level in different way with degree. The information might be useful for understanding the meaning the each system of traditional acupuncture therapy developed in each country.

The present study also offers a useful information regarding issues of specific effect of acupuncture. Recently a society of researchers of acupuncture (Society of Acupuncture Research: SAR) reported a white paper on future acupuncture research in the next decade [32]. One of the important issues of the SAR white paper is to explain the results obtained by well-designed acupuncture clinical trials conducted in USA [33]. The purpose of the RCT was to clarify the specific effect of acupuncture and significance of individualized TCM point selection. The obtained results indicated no statistically significant differences among the individualized TCM treatment, standardized TCM treatment and simulated acupuncture treatment (the skin was stimulated by tooth pick), although they were significantly more effective than Western standard therapy [33].

One simple explanation is to assume these acupuncture treatments are strong placebo effects, and no specific effect exists. The comparison of TCM acupuncture and simulated acupuncture with tooth pick seems to be reasonable protocol to clarify the specific effect of acupuncture, however the present study clearly demonstrated that TCM acupuncture is one of acupuncture procedures and other procedures developed in Japan and Korea also have modulatory effects on bio-regulatory systems. So simulated acupuncture, it might be included as real acupuncture technique in Japan, could not assume an inert sham intervention. So it is keenly required to develop inert sham acupuncture device or technique to clarify the existence of specific effects of acupuncture.

It should be noted that this project had several limitations. The major issue is the choice of subjects. The initial protocol was planned to select the subjects from three countries, but the time schedule of practitioners could not allow the selection from three countries, and small number of Japanese young male students who have no experience of acupuncture could be prepared. The selection of subjects in three countries might be one important issue in the future project. The selection of traditional acupuncture practitioners was also important issue. In Japan a practitioner who participated in the previous study [17] was adopted as key member of this project. In Korea, members of the Korean Acupuncture and Moxibustion Society (KAMS) discussed and recommended the practitioner in this study. In Chinese acupuncture, one Chinese acupuncturist who has acupuncture clinic in Japan was adopted. The individual variation of diagnosis of TCM doctors was pointed out [3], so it is important to prepare several practitioners in each country for obtaining sufficient and representative results of each country.

The feature of the present study was to examine three different traditional acupuncture treatments by applying 
to the same subjects. So crossover design was used. However the remarkable variation of baseline data and lack of control subjects, who received no acupuncture treatments, made it difficult to analyze and discuss the data sufficiently.

This clinical study of JCK traditional acupuncture was the first attempt in the world and it offered important information to establish the role of acupuncture therapy in CAM as an individualized therapy, and also offer interesting information for the future researches.

\section{References}

[1] Anastasi, J.K., Currie, L.M. and Kim, G.H. (2009) Understanding Diagnostic Reasoning in TCM Practice: Tongue Diagnosis. Alternative Therapies in Health and Medicine, 15, 18-28.

[2] Coyle, M. and Smith, C. (2005) A Survey Comparing TCM Diagnosis, Health Status and Medical Diagnosis in Women Undergoing Assisted Reproduction. Acupuncture Medicine, 23, 62-69. http://dx.doi.org/10.1136/aim.23.2.62

[3] Hogeboom, C.J., Sherman, K.J. and Cherkin, D.C. (2001) Variation in Diagnosis and Treatment of Chronic Low Back Pain by Traditional Chinese Medicine Acupuncturists. Complementary Therapies in Medicine, 9, 154-166. http://dx.doi.org/10.1054/ctim.2001.0457

[4] Zhang, G.G., Lee, W., Bausell, B., Lao, L., Handwerger, B. and Berman, B. (2005) Variability in the Traditional Chinese medicine (TCM) diagnoses and herbal prescriptions provided by three TCM practitioners for 40 patients with rheumatoid Arthritis. Journal of Alternative and Complementary Medicine, 11, 415-421. http://dx.doi.org/10.1089/acm.2005.11.415

[5] Lee, S.W., Jang, E.S., Lee, J. and Kim, J.Y. (2006) Current Researches on the Methods of Diagnosing Sasang Constitution: An Overview. Evidence-Based Complementary and Alternative Medicine, 6, 43-49. http://dx.doi.org/10.1093/ecam/nep092

[6] Kim, Y.S., Jun, H., Chae, Y., Park, H.J., Kim, B.H., Chang, I.M., et al. (2005) The Practice of Korean Medichine: An Overview of Clinical Trials in Acupuncture. Evidence-Based Complementary and Alternative Medicine, 2, 325-352. http://dx.doi.org/10.1093/ecam/neh102

[7] Kim, J.Y. and Pham, D.D. (2009) Sasang Constitutional Medicine as a Holistic Tailored Medicine. Evidence-Based Complementary and Alternative Medicine, 6, 11-19. http://dx.doi.org/10.1093/ecam/nep100

[8] Yoo, J.H., Kim, J.W., Kim, K.K., Kim, J.Y., Koh, B.H. and Lee, E.J. (2007) Sasangin Diagnosis Questionnaire: Test of Reliability. Journal of Alternative and Complementary Medicine, 13, 111-122. http://dx.doi.org/10.1089/acm.2006.5293

[9] Kobayashi, A., Uefuji, M. and Yasumo, W. (2010) History and Progress of Japanese Acupuncture. Evidence-Based Complementary and Alternative Medicine, 7, 359-365. http://dx.doi.org/10.1093/ecam/nem155

[10] Kawakita, K., Shichidou, T., Inoue, E., Nabeta, T., Kitakoji, H., Aizawa, S., et al. (2008) Do Japanese Style Acupuncture and Moxibustion Reduce Symptoms of the Common Cold? Evidence-Based Complementary and Alternative Medicine, 5, 481-489. http://dx.doi.org/10.1093/ecam/nem055

[11] Birch, S. and Jamison, R.N. (1998) Controlled Trial of Japanese Acupuncture for Chronic Myofascial Neck Pain: Assessment of Specific and Nonspecific Effects of Treatment. The Clinical Journal of Pain, 14, 248-255. http://dx.doi.org/10.1097/00002508-199809000-00012

[12] Kawakita, K., Shichidou, T., Inoue, E., Nabeta, T., Kitakouji, H., Aizawa, S., et al. (2004) Preventive and Curative Effects of Acupuncture on the Common Cold. A Multicentre Randomized Controlled Trial in Japan. Complementary Therapies in Medicine, 12, 181-188. http://dx.doi.org/10.1016/j.ctim.2004.10.004

[13] Witt, C., Brinkhaus, B., Jena, S., Linde, K., Streng, A., Wagenpfeil, S., et al. (2005) Acupuncture in Patients with Osteoarthritis of the Knee: A Randomised Trial. Lancet, 366, 136-143. http://dx.doi.org/10.1016/S0140-6736(05)66871-7

[14] Cummings, M. (2009) Modellvorhaben Akupunktur-A Summary of the ART, ARC and GERAC Trials. Acupuncture in Medicine, 27, 26-30. http://dx.doi.org/10.1136/aim.2008.000281

[15] Witt, C.M. and Brinkhaus, B. (2010) Efficacy, Effectiveness and Cost-Effectiveness of Acupuncture for Allergic Rhinitis-An Overview about Previous and Ongoing Studies. Autonomic Neuroscience, 157, 42-45. http://dx.doi.org/10.1016/j.autneu.2010.06.006

[16] WHO (2008) WHO Standard Acupuncture Point Locations in the Western Pacific Region. WPRO Nonserial Publication, Acpuncture Point Location.

[17] Yamaguchi, N., Takahashi, T., Sakuma, M., Sugita, T., Uchikawa, K., Sakaihara, S., et al. (2007) Acupuncture Regulates Leukocyte Subpopulations in Human Peripheral Blood. Evidence-Based Complementary and Alternative Medicine, 4, 447-453. http://dx.doi.org/10.1093/ecam/nel107 
[18] Abo, T., Kawate, T., Itoh, K. and Kumagai, K. (1988) Studies on the Bioperiodicity of the Immune Response. I. Circadian Rhythms of Human T, B and K Cell Traffic in the Peripheral Blood. The Journal of Immunology, 126, 1360-1363.

[19] Evans, D.L., Pedersen, C.A. and Folds, J.D. (1988) Major Depression and Immunity-Preliminary Evidence of Decreased Natural Killer Cell Populations. Progress in Neuro-Psychopharmacology and Biological Psychiatry, 12, 739748. http://dx.doi.org/10.1016/0278-5846(88)90019-X

[20] Arranz, L., Guayerbas, N., Siboni, L. and De la Fuente, M. (2007) Effect of Acupuncture Treatment on the Immune Function Impairment Found in Anxious Women. The American Journal of Chinese Medicine, 35, 35-51. http://dx.doi.org/10.1142/S0192415X07004606

[21] Kavoussi, B. and Ross, B.E. (2007) The Neuroimmune Basis of Anti-Inflammatory Acupuncture. Integrative Cancer Therapies, 6, 251-257. http://dx.doi.org/10.1177/1534735407305892

[22] Yamashita, H., Ichiman, Y. and Tanno, Y. (2001) Changes in Peripheral Lymphocyte Subpopulations after Direct Moxibustion. The American Journal of Chinese Medicine, 29, 227-235. http://dx.doi.org/10.1142/S0192415X01000265

[23] Kho, H.G., Van Egmond, J., Eijk, R.J. and Kapteyns, W.M. (1991) Lack of Influence of Acupuncture and Transcutaneous Stimulation on the Immunoglobulin Levels and Leukocyte Counts Following Upper-Abdominal Surgery. European Journal of Anaesthesiology, 8, 39-45.

[24] Kim, C.K., Choi, G.S., Oh, S.D., Han, J.B., Kim, S.K., Ahn, H.J., et al. (2005) Identification of Genes Altering Their Expressions in Electroacupuncture Induced Up-Regulation of Natural Killer Cell Activity. Journal of Neuroimmunology, 168, 144-153.

[25] Wang, J., Zhao, H., Mao-Ying, Q.L., Cao, X.D., Wang, Y.Q. and Wu, G.C. (2009) Electroacupuncture Downregulates TLR2/4 and Pro-Inflammatory Cytokine Expression after Surgical Trauma Stress without Adrenal Glands Involvement. Brain Research Bulletin, 80, 89-94. http://dx.doi.org/10.1016/j.brainresbull.2009.04.020

[26] Yu, Y., Kasahara, T., Sato, T., Guo, S.Y., Liu, Y., Asano, K., et al. (1997) Enhancement of Splenic Interferon-Gamma, Interleukin-2, and NK Cytotoxicity by S36 Acupoint Acupuncture in F344 Rats. The Japanese Journal of Physiology, 47, 173-178. http://dx.doi.org/10.2170/jjphysiol.47.173

[27] Yu, Y., Kasahara, T., Sato, T., Asano, K., Yu, G., Fang, J., et al. (1998) Role of Endogenous Interferon-Gamma on the Enhancement of Splenic NK Cell Activity by Electroacupuncture Stimulation in Mice. Journal of Neuroimmunology, 90, 176-186. http://dx.doi.org/10.1016/S0165-5728(98)00143-X

[28] Sato, T., Yu, Y., Guo, S.Y., Kasahara, T. and Hisamitsu, T. (1996) Acupuncture Stimulation Enhances Splenic Natural Killer Cell Cytotoxicity in Rats. The Japanese Journal of Physiology, 46, 131-136. http://dx.doi.org/10.2170/jiphysiol.46.131

[29] Ma, Z., Wang, Y. and Fan, Q. (1992) The Influence of Acupuncture on Interleukin 2 Interferon-Natural Killer Cell Regulatory Network of Kidney-Deficiency Mice. In Contact with Professor Kawakita K., 17, 139-142.

[30] Toyabe, S., Iiai, T., Fukuda, M., Kawamura, T., Suzuki, S., Uchiyama, M., et al. (1997) Identification of Nicotinic Acetylcholine Receptors on Lymphocytes in the Periphery as Well as Thymus in Mice. Immunology, 92, 201-205. http://dx.doi.org/10.1046/j.1365-2567.1997.00323.x

[31] Abo, T. (2003) Immune Revolution. Kohdansha International Publisher, Tokyo. (in Japanese)

[32] Langevin, H.M., Wayne, P.M., Macpherson, H., Schnyer, R., Milley, R.M., Napadow, V., et al. (2011) Paradoxes in Acupuncture Research: Strategies for Moving Forward. Evidence-Based Complementary and Alternative Medicine, 2011, Article ID: 180805. http://dx.doi.org/10.1155/2011/180805

[33] Cherkin, D.C., Sherman, K.J., Avins, A.L., Erro, J.H., Ichikawa, L., Barlow, W.E., et al. (2009) A Randomized Trial Comparing Acupuncture, Simulated Acupuncture, and Usual Care for Chronic Low Back Pain. Archives of Internal Medicine, 169, 858-866. http://dx.doi.org/10.1001/archinternmed.2009.65 